\title{
Harmonic mappings for which co-analytic part is a close-to-convex function of order $b$
}

\author{
Yaşar Polatog̃lu', Yasemin Kahramaner ${ }^{2}$ and Melike Aydogan ${ }^{3^{*}}$
}

${ }^{\text {*Correspondence: }}$

melike.aydogan@isikun.edu.tr

${ }^{3}$ Department of Mathematics, Isik

University, Sile Campus, İstanbul,

Turkey

Full list of author information is

available at the end of the article

\begin{abstract}
In the present paper we investigate a class of harmonic mappings for which the second dilatation is a close-to-convex function of complex order $b, b \in \mathbb{C} /\{0\}$ (Lashin in Indian J. Pure Appl. Math. 34(7):1101-1108, 2003).
\end{abstract}

MSC: $30 C 45 ; 30 C 55$

Keywords: harmonic mappings; complex dilatation; distortion theorem; growth theorem

\section{Introduction}

A planar harmonic mapping in the open unit disc $\mathbb{D}=\{z|| z \mid<1\}$ is a complex-valued harmonic function $f$ which maps $\mathbb{D}$ onto some planar domain $f(\mathbb{D})$. Since $\mathbb{D}$ is a simply connected domain, the mapping $f$ has a canonical decomposition $f=h(z)+\overline{g(z)}$, where $h(z)$ and $g(z)$ are analytic in $\mathbb{D}$ and have the following power series expansions:

$$
h(z)=\sum_{n=0}^{\infty} a_{n} z^{n}, \quad g(z)=\sum_{n=0}^{\infty} b_{n} z^{n}, \quad z \in \mathbb{D}
$$

where $a_{n}, b_{n} \in \mathbb{C}, n=0,1,2, \ldots$ As usual, we call $h(z)$ analytic part and $g(z)$ co-analytic part of $f$, respectively. An elegant and complete account of the theory of planar harmonic mappings is given in Duren?s monograph $\mathbb{1}]$.

Lewy [2] proved in 1936 that the harmonic mapping $f$ is locally univalent in $\mathbb{D}$ if and only if its Jacobian $J_{f}=\left|h^{\prime}(z)\right|^{2}-\left|g^{\prime}(z)\right|^{2}$ is different from zero in $\mathbb{D}$. In view of this result, locally univalent harmonic mappings in the open unit disc are either sensereversing if $\left|g^{\prime}(z)\right|>\left|h^{\prime}(z)\right|$ or sense-preserving if $\left|g^{\prime}(z)\right|<\left|h^{\prime}(z)\right|$ in $\mathbb{D}$. Throughout this paper, we restrict ourselves to the study of sense-preserving harmonic mappings. We also note that $f=h(z)+\overline{g(z)}$ is sense-preserving in $\mathbb{D}$ if and only if $h^{\prime}(z)$ does not vanish in the unit disc $\mathbb{D}$, and the second dilatation $w(z)=g^{\prime}(z) / h^{\prime}(z)$ has the property $|w(z)|<1$ in $\mathbb{D}$.

The class of all sense-preserving harmonic mappings in the open unit disc $\mathbb{D}$ with $a_{0}=$ $b_{0}=0$ and $a_{1}=1$ is denoted by $\mathcal{S}_{\mathcal{H}}$. Thus $\mathcal{S}_{\mathcal{H}}$ contains the standard class $\mathcal{S}$ of analytic univalent functions.

The family of all mappings $f \in \mathcal{S}_{\mathcal{H}}$ with the additional property that $g^{\prime}(0)=0$, i.e., $b_{1}=0$, is denoted by $\mathcal{S}_{\mathcal{H}}^{0}$. Thus it is clear that $\mathcal{S} \subset \mathcal{S}_{\mathcal{H}}^{0} \subset \mathcal{S}_{\mathcal{H}}$ [1]. Let $\Omega$ be the family of functions $\phi(z)$ regular in the open unit disc $\mathbb{D}$ and satisfying the conditions $\phi(0)=0,|\phi(z)|<1$ for all

\section{Springer}

(c) 2015 Polatog̃lu et al.: licensee Springer. This is an Open Access article distributed under the terms of the Creative Commons Attribution License (http://creativecommons.org/licenses/by/4.0), which permits unrestricted use, distribution, and reproduction in any medium, provided the original work is properly credited. 
$z \in \mathbb{D}$. We denote by $\mathcal{P}$ the family of functions $p(z)=1+p_{1} z+p_{2} z^{2}+\cdots$ regular in $\mathbb{D}$ such that $p(z)$ in $\mathcal{P}$ if and only if

$$
p(z)=\frac{1+\phi(z)}{1-\phi(z)}
$$

for some $\phi(z) \in \Omega$ and every $z \in \mathbb{D}$.

Let $s_{1}(z)=z+c_{2} z^{2}+c_{3} z^{3}+\cdots$ and $s_{2}(z)=z+d_{2} z^{2}+d_{3} z^{3}+\cdots$ be analytic functions in $\mathbb{D}$. If there exists a function $\phi(z) \in \Omega$ such that $s_{1}(z)=s_{2}(\phi(z))$ for every $z \in \mathbb{D}$, then we say that $s_{1}(z)$ is subordinate to $s_{2}(z)$ and we write $s_{1} \prec s_{2}$. We also note that if $s_{1} \prec s_{2}$, then $s_{1}(\mathbb{D}) \subset s_{2}(\mathbb{D})[3,4]$.

Next, let $\mathcal{A}$ be the class of functions $s(z)=z+e_{2} z^{2}+\cdots$ which are analytic in $\mathbb{D}$. A function $s(z)$ in $\mathcal{A}$ is said to be a convex function of complex order $b, b \in \mathbb{C} /\{0\}$, that is, $s(z) \in \mathcal{C}(b)$ if and only if $s^{\prime}(z) \neq 0$, and

$$
\operatorname{Re}\left(1+\frac{1}{b} z \frac{s^{\prime \prime}(z)}{s^{\prime}(z)}\right)>0 \quad(z \in \mathbb{D})
$$

We denote by $\mathcal{S}^{*}(1-b)$ the class of $\mathcal{A}$ consisting of functions which are starlike of complex order $b$, that is,

$$
\operatorname{Re}\left(1+\frac{1}{b}\left(z \frac{s^{\prime \prime}(z)}{s^{\prime}(z)}-1\right)\right)>0 \quad(z \in \mathbb{D})
$$

Moreover, let $s(z)$ be an element of $\mathcal{A}$, then $s(z)$ is said to be close-to-convex of complex order $b, b \in \mathbb{C} /\{0\}$ if and only if there exists a function $\varphi(z) \in \mathcal{C}(b)$ satisfying the condition

$$
\operatorname{Re}\left(1+\frac{1}{b}\left(\frac{s^{\prime}(z)}{\varphi^{\prime}(z)}-1\right)\right)>0 \quad(z \in \mathbb{D})
$$

The class of such functions is denoted by $\mathcal{C C}(b)$.

The classes $\mathcal{C}(b)$ and $\mathcal{S}^{*}(1-b)$ were introduced and studied by Nasr and Aouf [5, 6], and the class $\mathcal{C C}(b)$ was introduced by Lashin [7].

\section{Remark 1.1}

(i) For $b=1$ we obtain $\mathcal{S}^{*}(0)=\mathcal{S}^{*}, \mathcal{C}(1)=\mathcal{C}$, and $\mathcal{C C}(1)=\mathcal{C C}$ are well-known classes of starlike, convex and close-to-convex functions, respectively [6].

(ii) $\mathcal{S}^{*}(1-(1-\alpha))=\mathcal{S}^{*}(\alpha), \mathcal{C}(1-\alpha)$, and $\mathcal{C C}(1-\alpha), 0 \leq \alpha<1$, are the classes of starlike, convex and close-to-convex functions of order $\alpha$, respectively [6].

(iii) If we take $b=e^{-i \lambda} \cos \lambda,|\lambda|<\pi / 2$, we obtain the following classes: $\lambda$-spirallike, analytic functions for which $z f^{\prime}(z)$ is $\lambda$-spirallike and $\lambda$-spirallike and $\lambda$-spiral close-to-convex functions [6].

(iv) $\mathcal{S}^{*}\left(1-(1-\alpha) e^{-i \lambda} \cos \lambda\right), \mathcal{C}^{*}\left((1-\alpha) e^{-i \lambda} \cos \lambda\right), \mathcal{C C}^{*}\left((1-\alpha) e^{-i \lambda} \cos \lambda\right), 0 \leq \alpha<1$, $|\lambda|<\pi / 2$, are the classes of $\lambda$-spirallike functions of order $\alpha$, analytic functions for which $z f^{\prime}(z)$ is $\lambda$-spirallike of order $\alpha$ and $\lambda$-spiral close-to-convex functions of order $\alpha$, respectively [6]. 
Finally, the aim of this investigation is to obtain some properties of the class of harmonic functions defined by

$$
\begin{aligned}
\mathcal{S}_{\mathcal{H C C}(b)=} & \left\{f=h(z)+\overline{g(z)} \mid w(z)=\frac{g^{\prime}(z)}{h^{\prime}(z)} \prec b_{1} \frac{1+(2 b-1) z}{1-z}\right. \\
& \left.\Leftrightarrow \operatorname{Re}\left[1+\frac{1}{b}\left(\frac{g^{\prime}(z)}{h^{\prime}(z)}-b_{1}\right)\right]>0, b, b_{1} \in \mathbb{C} /\{0\}, h(z) \in \mathcal{C}(b)\right\}
\end{aligned}
$$

for all $z$ in $\mathbb{D}$.

For the purpose of this paper, we need the following lemma and theorem.

Lemma 1.2 [8] Let $\phi(z)$ be regular in the unit disc $\mathbb{D}$ with $\phi(0)=0$. If the maximum value of $|\phi(z)|$ on the circle $|z|=r<1$ is attained at point $z_{1}$, then we have $z_{1} \phi^{\prime}\left(z_{1}\right)=k \phi\left(z_{1}\right)$ for some $k \geq 1$.

Theorem 1.3 [9] If $s(z) \in \mathcal{C}(b)$, then

$$
2\left[1+\frac{1}{b}\left(z \frac{s^{\prime}(z)}{s(z)}-1\right)\right]-1=p(z)=\frac{1+\phi(z)}{1-\phi(z)}
$$

for some $\phi(z) \in \Omega$ and every $z$ in $\mathbb{D}$, and

$$
\int_{0}^{2 \pi} \operatorname{Re}\left(z \frac{s^{\prime}(z)}{s(z)}\right) d \theta=2 p n \pi
$$

for every $z \in \mathbb{D}$. A member of $\mathcal{S}^{*}(p, n)$ is called p-valent starlike function in the unit disc $\mathbb{D}$.

Finally, a planar harmonic mapping in the open unit disc $\mathbb{D}$ is a complex-valued harmonic function $f$, which maps $\mathbb{D}$ onto some planar domain $f(\mathbb{D})$. Since $\mathbb{D}$ is a simply connected domain, the mapping $f$ has a canonical decomposition $f=h+\bar{g}$, where $h(z)$ and $g(z)$ are analytic in $\mathbb{D}$ and have the following power series expansion:

$$
h(z)=z^{p}+a_{n p+1} z^{n p+1}+a_{n p+2} z^{n p+2}+\cdots+a_{n p+m} z^{n p+m}+\cdots
$$

and

$$
g(z)=b_{n p} z^{n p}+b_{n p+1} z^{n p+1}+b_{n p+2} z^{n p+2}+\cdots+b_{n p+m} z^{n p+m}+\cdots,
$$

where $\left|b_{n p}\right|<1, p \geq 1$ and $n \geq 1$ are integers, $a_{n p+m}, b_{n p+m} \in \mathbb{C}$ and every $z \in \mathbb{D}$. As usual, we call $h(z)$ the analytic part and $g(z)$ the co-analytic part of $f$, respectively, and let the class of such harmonic mappings be denoted by $\mathcal{S H}(p, n)$. Lewy [2] proved in 1936 that the harmonic mapping $f$ is locally univalent in $\mathbb{D}$ if and only if its Jacobian $J_{f}=\left|h^{\prime}(z)\right|^{2}-\left|g^{\prime}(z)\right|^{2}$ is strictly positive in $\mathbb{D}$. In view of this result, locally univalent harmonic mappings in the open unit disc are either sense-reversing if $\left|g^{\prime}(z)\right|>\left|h^{\prime}(z)\right|$ or sense-preserving if $\left|g^{\prime}(z)\right|<$ $\left|h^{\prime}(z)\right|$ in $\mathbb{D}$. Throughout this paper, we restrict ourselves to the study of sense-preserving harmonic mappings. We also note that an elegant and complete treatment theory of the harmonic mapping is given in Duren?s monograph $\mathbb{1}]$. 
The main aim of this paper is to investigate some properties of the following class:

$$
\begin{aligned}
\mathcal{S}^{*} \mathcal{H}(p, n)= & \left\{f=h+\bar{g} \in \mathcal{S H}(p, n) \mid w(z)=\frac{g^{\prime}(z)}{h^{\prime}(z)} \prec b_{n p} \frac{1+\phi(z)}{1-\phi(z)},\right. \\
& \left.\phi(z)=z^{n} \psi(z), \psi(z) \in \Omega_{1}, h(z) \in \mathcal{S}^{*}(p, n), z \in \mathbb{D}\right\}
\end{aligned}
$$

and for this aim we need the following lemma.

Lemma 1.4 [1] Let $w(z)=a_{n} z^{n}+a_{n+1} z^{n+1}+a_{n+1} z^{n+2}+\cdots\left(a_{n} \neq 0, n \geq 1\right)$ be analytic in $\mathbb{D}$. If the maximum value of $|w(z)|$ on the circle $|z|=r<1$ is attained at $z=z_{0}$, then we have $z_{0} w^{\prime}\left(z_{0}\right)=p w\left(z_{0}\right)$, where $p \geq n$ and every $z \in \mathbb{D}$.

\section{Main results}

Lemma 2.1 Let $h(z)$ be an element of $\mathcal{C}(b)$, then

$$
\mathcal{F}_{1}\left(\frac{1}{2}|b|, \frac{1}{2} \operatorname{Re} b,-r\right) \leq|h(z)| \leq \mathcal{F}_{1}\left(\frac{1}{2}|b|, \frac{1}{2} \operatorname{Re} b, r\right)
$$

and

$$
\mathcal{F}_{2}(|b|, \operatorname{Re} b,-r) \leq\left|h^{\prime}(z)\right| \leq \mathcal{F}_{2}(|b|, \operatorname{Re} b, r)
$$

where

$$
\mathcal{F}_{1}\left(\frac{1}{2}|b|, \frac{1}{2} \operatorname{Re} b,-r\right)=\frac{(1+r)^{|b|-\operatorname{Re} b}}{(1-r)^{|b|+\operatorname{Re} b}}
$$

and

$$
\mathcal{F}_{2}(|b|, \operatorname{Re} b, r)=\frac{(1+r)^{\frac{1}{2}|b|-\frac{1}{2} \operatorname{Re} b}}{(1-r)^{\frac{1}{2}|b|+\frac{1}{2} \operatorname{Re} b}} .
$$

These inequalities are sharp because the extremal function is $h(z)=\frac{1}{(1-z)^{b}}$ with $z=\frac{r\left(r-\frac{\bar{b}}{b}\right)^{1 / 2}}{1-r\left(\frac{b}{b}\right)^{1 / 2}}$.

Proof Using Theorem 1.3, the definition of class $\mathcal{C}(b)$ and the definition of the subordination principle, we obtain

$$
z \frac{h^{\prime}(z)}{h(z)}=\frac{1+(b-1) \phi(z)}{1-\phi(z)} \Rightarrow z \frac{h^{\prime}(z)}{h(z)} \prec \frac{1+(b-1) z}{1-z}
$$

or

$$
\left|z \frac{h^{\prime}(z)}{h(z)}-\frac{b r^{2}}{1-r^{2}}\right| \leq \frac{|b| r}{1-r^{2}}
$$

and similarly

$$
z \frac{h^{\prime \prime}(z)}{h^{\prime}(z)}=\frac{2 b \phi(z)}{1-\phi(z)} \quad \Rightarrow \quad z \frac{h^{\prime \prime}(z)}{h^{\prime}(z)} \prec \frac{2 b z}{1-z}
$$


or

$$
\left|z \frac{h^{\prime \prime}(z)}{h^{\prime}(z)}-\frac{2 b r^{2}}{1-r^{2}}\right| \leq \frac{2|b| r}{1-r^{2}}
$$

Using (2.5) and (2.6), we get (2.1) and (2.2), respectively.

Theorem 2.2 Let $f=h(z)+\overline{g(z)}$ be an element of $\mathcal{S}_{\mathcal{H C C}(b)}$, then

$$
\frac{g(z)}{h(z)} \prec b_{1} \frac{1+(2 b-1) z}{1-z} \quad(z \in \mathbb{D})
$$

Proof Since $f=h(z)+\overline{g(z)}$ is an element of $\mathcal{S}_{\mathcal{H C C}(b)}$, then we have

$$
\frac{g^{\prime}(z)}{h^{\prime}(z)} \prec b_{1} \frac{1+(2 b-1) z}{1-z} \Leftrightarrow \operatorname{Re}\left[1+\frac{1}{b}\left(\frac{g^{\prime}(z)}{h^{\prime}(z)}-b_{1}\right)\right]>0,
$$

so

$$
\frac{g^{\prime}(z)}{h^{\prime}(z)}=b_{1} \frac{1+(2 b-1) \phi(z)}{1-\phi(z)}
$$

for some $\phi(z) \in \Omega$ and every $z$ in $\mathbb{D}$. Now, we define the function $\phi(z)$ by

$$
\frac{g(z)}{h(z)}=b_{1} \frac{1+\phi(z)}{1-\phi(z)} \quad(z \in \mathbb{D})
$$

then $\phi(z)$ is analytic in $\mathbb{D}$ and $\left.\frac{g(z)}{h(z)}\right|_{z=0}=b_{1}=b_{1} \frac{1+\phi(0)}{1-\phi(0)}$, then $\phi(0)=0$ and

$$
w(z)=\frac{g^{\prime}(z)}{h^{\prime}(z)}=b_{1}\left(\frac{1+\phi(z)}{1-\phi(z)}+\frac{2 z \phi(z)}{1-\phi(z)} \cdot \frac{1}{1+(b-1) \phi(z)}\right) \quad(z \in \mathbb{D}) .
$$

Now it is easy to realize that the subordination $\frac{g^{\prime}(z)}{h^{\prime}(z)} \prec b_{1} \frac{1+(2 b-1) z}{1-z}$ is equivalent to $|\phi(z)|<1$ for all $z \in \mathbb{D}$. Indeed, assume to the contrary, that there exists $z_{1} \in \mathbb{D}$ such that $\left|\phi\left(z_{1}\right)\right|=1$. Then by Jack?s lemma (Lemma1.4), $z_{1} \phi^{\prime}\left(z_{1}\right)=k \phi\left(z_{1}\right), k \geq 1$, for such $z_{1} \in \mathbb{D}$, we have

$$
w\left(z_{1}\right)=\frac{g^{\prime}\left(z_{1}\right)}{h^{\prime}\left(z_{1}\right)}=b_{1}\left(\frac{1+\phi\left(z_{1}\right)}{1-\phi\left(z_{1}\right)}+\frac{2 k \phi\left(z_{1}\right)}{1-\phi\left(z_{1}\right)} \cdot \frac{1}{1+(b-1) \phi\left(z_{1}\right)}\right)=w\left(\phi\left(z_{1}\right)\right) \notin w(\mathbb{D})
$$

because $\left|\phi\left(z_{1}\right)\right|=1$ and $k \geq 1$. But this is a contradiction to the condition $\frac{g^{\prime}(z)}{h^{\prime}(z)} \prec b_{1} \frac{1+(2 b-1) z}{1-z}$, and so assumption is wrong, i.e., $|\phi(z)|<1$ for all $z \in \mathbb{D}$.

Corollary 2.3 Let $f=h(z)+\overline{g(z)}$ be an element of $\mathcal{S}_{\mathcal{H C C}(b)}$, then

$$
\begin{aligned}
& \mathcal{F}_{1}\left(\frac{1}{2}|b|, \frac{1}{2} \operatorname{Re} b,-r\right) \frac{\left|b_{1}\right|-2|b| r-\left|b_{1}-2 b\right| r^{2}}{1-r^{2}} \\
& \quad \leq|g(z)| \leq \mathcal{F}_{1}\left(\frac{1}{2}|b|, \frac{1}{2} \operatorname{Re} b, r\right) \frac{\left|b_{1}\right|+2|b| r+\left|b_{1}-2 b\right| r^{2}}{1-r^{2}}
\end{aligned}
$$


and

$$
\begin{aligned}
& \mathcal{F}_{2}(|b|, \operatorname{Re} b,-r) \frac{\left|b_{1}\right|-2|b| r-\left|b_{1}-2 b\right| r^{2}}{1-r^{2}} \\
& \quad \leq\left|g^{\prime}(z)\right| \leq \mathcal{F}_{2}(|b|, \operatorname{Re} b, r) \frac{\left|b_{1}\right|+2|b| r+\left|b_{1}-2 b\right| r^{2}}{1-r^{2}}
\end{aligned}
$$

for all $|z|=r<1$, where $\mathcal{F}_{1}$ and $\mathcal{F}_{2}$ are defined by (2.3) and (2.4), respectively.

Proof Since $f=h(z)+\overline{g(z)} \in \mathcal{S}_{\mathcal{H C C}(b)}$, we have

$$
\operatorname{Re}\left[1+\frac{1}{b}\left(\frac{g^{\prime}(z)}{h^{\prime}(z)}-b_{1}\right)\right]>0 \Leftrightarrow \frac{g^{\prime}(z)}{h^{\prime}(z)} \prec b_{1} \frac{1+(2 b-1) z}{1-z}
$$

or

$$
\left|\frac{g^{\prime}(z)}{h^{\prime}(z)}-\frac{b_{1}+\left(2 b-b_{1}\right) r^{2}}{1-r^{2}}\right| \leq \frac{2|b| r}{1-r^{2}}
$$

then

$$
\frac{\left|b_{1}\right|-2|b| r-\left|b_{1}-2 b\right| r^{2}}{1-r^{2}} \leq \frac{\left|g^{\prime}(z)\right|}{\left|h^{\prime}(z)\right|} \leq \frac{\left|b_{1}\right|+2|b| r+\left|b_{1}-2 b\right| r^{2}}{1-r^{2}}
$$

and using Theorem 2.2 we obtain

$$
\left|\frac{g(z)}{h(z)}-\frac{b_{1}+\left(2 b-b_{1}\right) r^{2}}{1-r^{2}}\right| \leq \frac{2|b|}{1-r^{2}}
$$

or

$$
\frac{\left|b_{1}\right|-2|b| r-\left|b_{1}-2 b\right| r^{2}}{1-r^{2}} \leq \frac{|g(z)|}{|h(z)|} \leq \frac{\left|b_{1}\right|+2|b| r+\left|b_{1}-2 b\right| r^{2}}{1-r^{2}}
$$

for all $|z|=r<1$. Considering Lemma 2.1, (2.10) and (2.11) together, we obtain (2.8) and (2.9).

Lemma 2.4 Iff $=h(z)+\overline{g(z)} \in \mathcal{S}_{\mathcal{H C C}(b)}$, then

$$
\begin{aligned}
& \frac{\left|b_{1}\right|-r}{1+\left|b_{1}\right| r} \leq|w(z)| \leq \frac{\left|b_{1}\right|+r}{1+\left|b_{1}\right| r}, \\
& \frac{\left(1-r^{2}\right)\left(1-\left|b_{1}\right|^{2}\right)}{\left(1+\left|b_{1}\right| r\right)^{2}} \leq 1-|w(z)|^{2} \leq \frac{\left(1-r^{2}\right)\left(1-\left|b_{1}\right|^{2}\right)}{\left(1-\left|b_{1}\right| r\right)^{2}}, \\
& \frac{(1-r)\left(1+\left|b_{1}\right|\right)}{1-\left|b_{1}\right| r} \leq 1+|w(z)| \leq \frac{(1+r)\left(1+\left|b_{1}\right|\right)}{1+\left|b_{1}\right| r}
\end{aligned}
$$

and

$$
\frac{(1-r)\left(1-\left|b_{1}\right|\right)}{1+\left|b_{1}\right| r} \leq 1-|w(z)| \leq \frac{(1+r)\left(1-\left|b_{1}\right|\right)}{1-\left|b_{1}\right| r}
$$

for all $|z|=r<1$. 
Proof Since $f=h(z)+\overline{g(z)} \in \mathcal{S}_{\mathcal{H C C}(b)}$, it follows that

$$
w(z)=\frac{g^{\prime}(z)}{h^{\prime}(z)}=\frac{b_{1}+2 b_{2} z+\cdots}{1+2 a_{2} z+\cdots} \quad \text { so } w(0)=b_{1} \text { and }|w(z)|<1 .
$$

So, the function

$$
\phi(z)=\frac{w(z)-w(0)}{1-\overline{w(0)} w(z)}=\frac{w(z)-b_{1}}{1-\bar{b}_{1} w(z)} \quad(z \in \mathbb{D})
$$

satisfies the conditions of Schwarz lemma. Therefore, we have

$$
w(z)=\frac{b_{1}+\phi(z)}{1+\bar{b}_{1} \phi(z)} \quad \text { if and only if } \quad w(z) \prec \frac{b_{1}+z}{1+\bar{b}_{1} z} \quad(z \in \mathbb{D}) \text {. }
$$

On the other hand, the linear transformation $\frac{b_{1}+z}{1+\bar{b}_{1} z}$ maps $|z|=r$ onto the disc with the center $C(r)=\left(\frac{\left(1-r^{2}\right) \operatorname{Re} b_{1}}{1-\left|b_{1}\right|^{2} r^{2}}, \frac{\left(1-r^{2}\right) \operatorname{Im} b_{1}}{1-\left|b_{1}\right|^{2} r^{2}}\right)$ and the radius $\rho(r)=\frac{\left(1-\left|b_{1}\right|^{2}\right) r}{1-\left|b_{1}\right| r^{2}}$. Then we have (2.12), which gives (2.13), (2.14) and (2.15).

Corollary 2.5 Let $f(z)$ be an element of $\mathcal{S}_{\mathcal{H C C}(b)}$, then

$$
\frac{\left(1-r^{2}\right)\left(1-\left|b_{1}\right|\right)^{2}}{\left(1+\left|b_{1}\right| r\right)^{2}}\left(\mathcal{F}_{2}(|b|, \operatorname{Re} b,-r)\right)^{2} \leq J_{f} \leq \frac{\left(1-r^{2}\right)\left(1-\left|b_{1}\right|\right)^{2}}{\left(1-\left|b_{1}\right| r\right)^{2}}\left(\mathcal{F}_{2}(|b|, \operatorname{Re} b, r)\right)^{2}
$$

and

$$
\begin{aligned}
& \left(1-\left|b_{1}\right|\right) \int_{0}^{r} \frac{1-\rho}{1+\left|b_{1}\right| \rho} \mathcal{F}_{2}(|b|, \operatorname{Re} b,-\rho) d \rho \\
& \quad \leq|f| \leq\left(1+\left|b_{1}\right|\right) \int_{0}^{r} \frac{1+\rho}{1+\left|b_{1}\right| \rho} \mathcal{F}_{2}(|b|, \operatorname{Re} b, \rho) d \rho
\end{aligned}
$$

for all $|z|=r<1$, where $\mathcal{F}_{2}$ is defined by (2.4).

Proof Since

$$
\left(1-|w(z)|^{2}\right)\left|h^{\prime}(z)\right|^{2} \leq J_{j} \leq\left(1+|w(z)|^{2}\right)\left|h^{\prime}(z)\right|
$$

and

$$
(1-|w(z)|)\left|h^{\prime}(z)\right||d z| \leq|d f| \leq(1+|w(z)|)\left|h^{\prime}(z)\right||d z|
$$

thus using Lemma 2.1 and Lemma 2.4 in the last two inequalities we obtain the desired result.

Theorem 2.6 Let $f(z)$ be an element of $\mathcal{S}_{\mathcal{H C C}(b)}$, then

$$
\sum_{k=2}^{n}\left|b_{k}-b_{1} a_{k}\right|^{2} \leq \sum_{k=1}^{n-1}\left|b_{k}+b_{1}(2 b-1) a_{k}\right|^{2}
$$


Proof Using Theorem 2.2, we obtain the following relation:

$$
\frac{g(z)}{h(z)} \prec b_{1} \frac{1+(2 b-1) z}{1-z} \Rightarrow \frac{g(z)}{h(z)}=\frac{b_{1}+b_{1}(2 b-1) \phi(z)}{1-\phi(z)}
$$

or

$$
g(z)-b_{1} h(z)=\left(g(z)+b_{1}(2 b-1) h(z)\right) \phi(z) \quad(z \in \mathbb{D}, \phi(z) \in \Omega) .
$$

Equality (2.16) can be written in the following form:

$$
\sum_{k=2}^{n}\left(b_{k}-b_{1} a_{k}\right) z^{k}+\sum_{k=n+1}^{\infty} d_{k} z^{k}=\left(\sum_{k=1}^{n-1}\left(b_{k}+b_{1}(2 b-1) a_{k}\right) z^{k}\right) \phi(z) \quad(z \in \mathbb{D}) .
$$

Since the last equality has the form $f_{1}(z)=f_{2}(z) \phi(z)$ with $|\phi(z)|<1$, it follows that

$$
\frac{1}{2 \pi} \int_{0}^{2 \pi}\left|f_{1}\left(r e^{i \theta}\right)\right|^{2} d \theta \leq \frac{1}{2 \pi} \int_{0}^{2 \pi}\left|f_{2}\left(r e^{i \theta}\right)\right|^{2} d \theta
$$

for each $r(0<r<1)$. Expressing $(2.18)$ in terms of the coefficients in $(2.17)$, we obtain the inequality

$$
\sum_{k=2}^{n}\left|b_{k}-b_{1} a_{k}\right|^{2} r^{2 k}+\sum_{k=n+1}^{\infty}\left|d_{k}\right|^{2} r^{2 k} \leq \sum_{k=1}^{n-1}\left|b_{k}+b_{1}(2 b-1) a_{k}\right|^{2} r^{2 k}
$$

where $d_{k}=\left(b_{k}-b_{1} a_{k}\right)-\left(b_{k}+b_{1}(2 b-1) a_{k}\right) \phi(z)$. By letting $r \rightarrow 1^{-}$in (2.19) we obtain the desired result. The proof of this method is due to Clunie [10].

\section{Competing interests}

The authors declare that they have no competing interests.

\section{Authors? contributions}

All authors contributed equally and significantly to writing this paper. All authors read and approved the final manuscript.

\section{Author details}

${ }^{1}$ Department of Mathematics and Computer Sciences, Istanbul Kültür University, İstanbul, Turkey. ${ }^{2}$ Department of Mathematics, Istanbul Commerce University, Üsküdar Campus, İstanbul, Turkey. ${ }^{3}$ Department of Mathematics, Isik University, Sile Campus, ístanbul, Turkey.

Received: 27 October 2014 Accepted: 22 December 2014 Published online: 16 January 2015

\section{References}

1. Duren, P: Harmonic Mappings in the Plane. Cambridge Tracts in Mathematics, vol. 156. Cambridge University Press, Cambridge (2004)

2. Lewy, H: On the non-vanishing of the Jacobian in certain in one-to-one mappings. Bull. Am. Math. Soc. 42, 689-692 (1936)

3. Goodman, AW: Univalent Functions, vol. I. Mariner Publishing, Tampa (1983)

4. Goodman, AW: Univalent Functions, vol. II. Mariner Publishing, Tampa (1983)

5. Nasr, MA, Aouf, MK: On convex functions of complex order. Mansoura Sci. Bull. 9, 565-582 (1982)

6. Nasr, MA, Aouf, MK: Starlike function of complex order. J. Nat. Sci. Math. 25(1), 1-12 (1985)

7. Lashin, AY: Starlike and convex functions of complex order involving a certain linear operator. Indian J. Pure Appl. Math. 34(7), 1101-1108 (2003)

8. Jack, IS: Functions starlike and convex of order $\alpha$. J. Lond. Math. Soc. (2) 3, 469-474 (1971)

9. Polatoğlu, Y, Bolcal, M, Sen, A: The radius of starlikeness for convex functions of complex order. Int. J. Math. Math. Sci. 2004(45), 2423-2428 (2004)

10. Clunie, J: On meromorphic schlicht functions. J. Lond. Math. Soc. 34, 215-216 (1959) 